\title{
Visualization of Superficial Cerebral Lesions Using a Smartphone Application
}

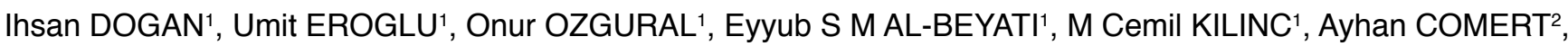 \\ Melih BOZKURT ${ }^{1}$ \\ ${ }^{1}$ Ankara University, School of Medicine, Department of Neurosurgery, Ankara, Turkey \\ ${ }^{2}$ Ankara University, School of Medicine, Department of Anatomy, Ankara, Turkey \\ This paper was presented orally in the $29^{\text {th }}$ Annual Scientific Congress of the Turkish Neurosurgical Society, 17-21 April, 2015, Antalya, Turkey.
}

\section{ABSTRACT}

AIM: Accurate localizations of cerebral lesions ensure good surgical outcomes and minimize intraoperative complications. Currently, microscope-integrated neuronavigation systems compensate these requirements. Since these units are expensive and large, the need to create more practical and portable systems in this "smart" century has become apparent.

MATERIAL and METHODS: The See-Through Camera smartphone application (app) is a photography app that penetrates the existing background picture and merges this with a camera image on the smartphone display. This app was applied to neurosurgery for visualization of the projection of the tumors over head in 20 patients. App accuracy was tested according to neuronavigation system.

RESULTS: Precision of the app was evaluated according to the superposition ratio of the tumor image drawn by the navigation system with the tumor picture on the smartphone display. This ratio was $40 \%-100 \%$ in 16 (80\%) patients and below $40 \%$ as an unfavorable result in the rest of the patients.

CONCLUSION: Basic rationality of this app can provide the basis of future pocket navigation systems in terms of its design and principles.

KEYWORDS: Application, Smartphone, Visualization, X-Ray

\section{INTRODUCTION}

A dvances in communication technology have transformed mobile phones into smartphones, which have become nearly indispensable in daily life and can function as portable pocket computers (14). The 'apps', which are applications specific to hand-held computers, including smartphones and tablets, have expanded the functions of mobile technology in social and business activities. In the medical field, many apps developed for use in health care, health education and health science are available for online purchase and downloading $(10,13,18)$. The benefits of these apps include mobile access to medical journals, treatment guidelines, one-touch glossaries, mobile measurement and monitoring of an individual's basic vital signs, guiding physicians in bedside management and assisting in preoperative evaluation of patients. $(2,3,7,9,11,12)$. Apps with specific medical uses have been developed for health care professionals and are available in online stores. However, non-medical apps are also available that can be adapted for use in the health sector $(6,8)$. Such apps, although currently unrelated to specific health sector areas, may expand the use of existing surgical apps in invasive procedures and illustrate basic principles of future mobile surgical programming apps. 
This report describes the adaptation of the See-Through Camera app to neurosurgery and its integration with surgical procedures. This app was designed for smartphones and tablet computers and transparently merges existing photographs as layers. It can be downloaded free from the Apple Store for use in iOS-based smartphones and tablets (https://itunes. apple.com/us/app/see-through-camera/id915347544?mt=8). It is beyond the scope of this article to discuss how to design future, hand-held neuronavigation systems.

\section{MATERIAL and METHODS}

\section{Patient Selection}

The study included 20 patients -8 men and 12 women-who had undergone neuronavigation-guided (BrainLab, Munich, Germany) transcranial surgery for superficial brain tumors located on the lateral cerebral convexities. All patients were assessed preoperatively by images obtained from the neuronavigation system. Surgical planning, including patient position, identification of the tumor location and scalp boundaries, the skin incision, and the size, shape, and re-implantation of the bone flap for the optimal surgical field, was conducted considering these data. Patient age ranged from 8 to 42 years. Preoperative surgical planning and preparation surgery were both entirely navigation assisted. Informed consent was obtained from patients.

\section{Procedures}

Drawing the tumor and bone flap figure on the scalp: The tumor location and boundaries and the designated bone flap to be created during craniotomy with guidance by the neuronavigation system were identified and clearly drawn on the scalp. The maximum estimated size of the tumor was drawn.

\section{Step-wise implementation of See-Through Camera app:}

Step 1: A recent magnetic resonance image that included the most lateral/superficial part of the tumor on sagittal section was initially displayed on-screen (Figure 1A).

Step 2: The border of the tumor was drawn in each sagittal slice from lateral (superficial) to medial (deep) (Figure 1A-E).

Step 3: The middle sagittal (main) image, the image passing through the sagittal sinus with the third ventricle, medial cerebral gyral anatomy, and the nose, was displayed on screen. Lateral views of the tumor boundaries and the midsagittal anatomic structures were merged with See-Through Camera app to form an image on a smartphone or tablet computer as shown in Figure 1F.

Step 4: A photograph of the patient was taken, and the picture was edited by touching the image on the screen. The region of the photo corresponding to the touched area of the display
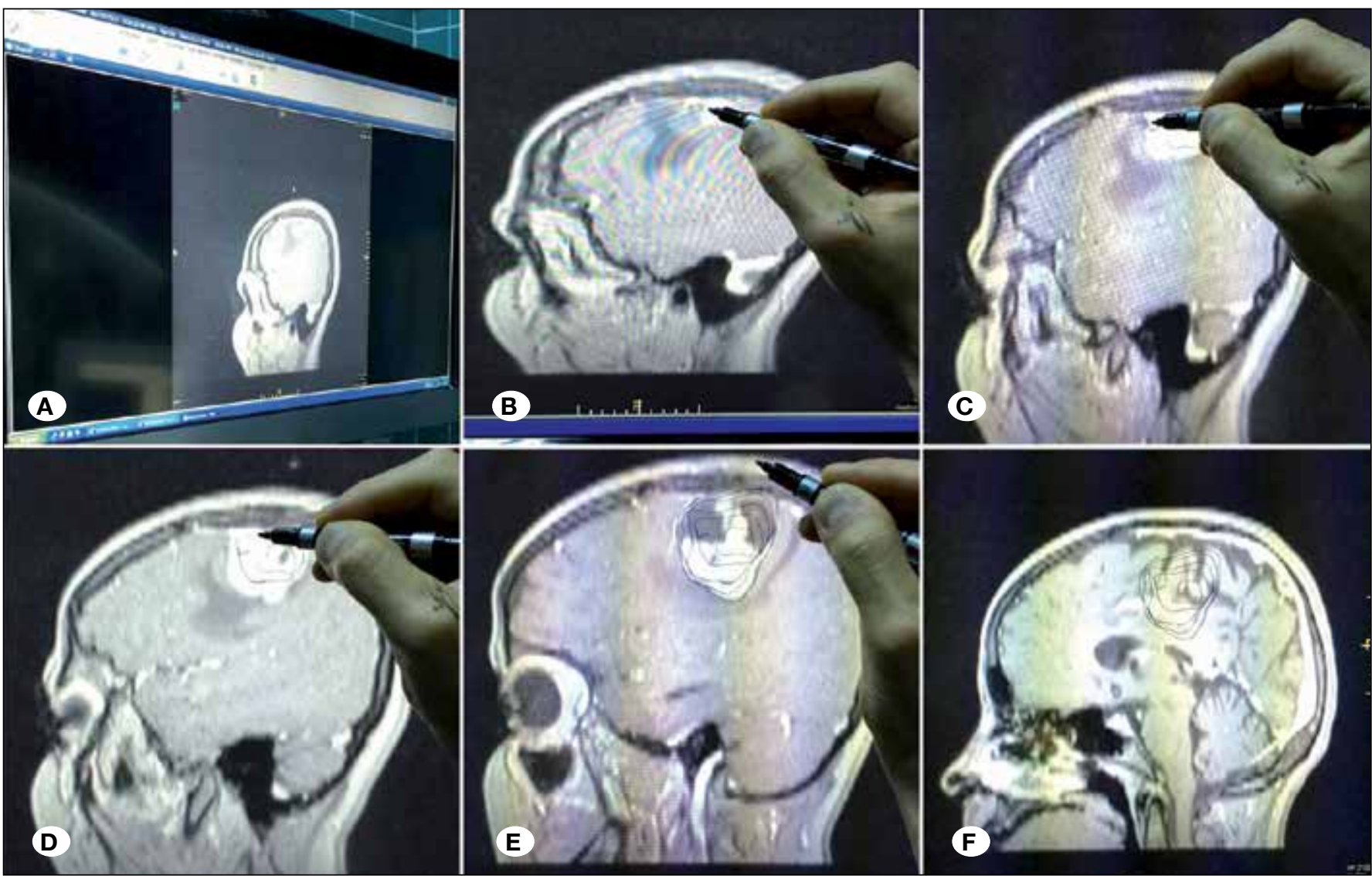

Figure 1: Step-wise drawing of tumor boundaries from lateral to medial sagittal MR images on the display (A-E) and creating the main image that includes the successive figure boundaries and the median sagittal image (F). 
was erased, and the resulting transparent area revealed the image of electronic device (Figure 2A-B). Editing the main image, while keeping visible (not touching) the line on the head surrounding the tumor, erased the area within the contour of the head and neck.

Step 5: The last step was fitting the contour of the camera image of patient to the transparent space within the appedited main image (Figure 2C-E). Some prominent landmarks such as the inion, nasion, and tip of nose should be when contour fitting. After image fusion and optimum display coupling, a new photo was taken of the magnetic resonance (MR) image and patient head (Figure 2F).

Comparison criteria and scoring: The superposition ratio of the tumor image (SRI) generated by See-Through Camera on the smartphone display and the schematic drawing of tumor inside the designated craniotomy bone flap figure was the primary criterion. Microscope-integrated neuronavigation systems have been approved for medical use and their accuracy has been confirmed. The See-Through Camera app was tested and compared with the BrainLab navigation
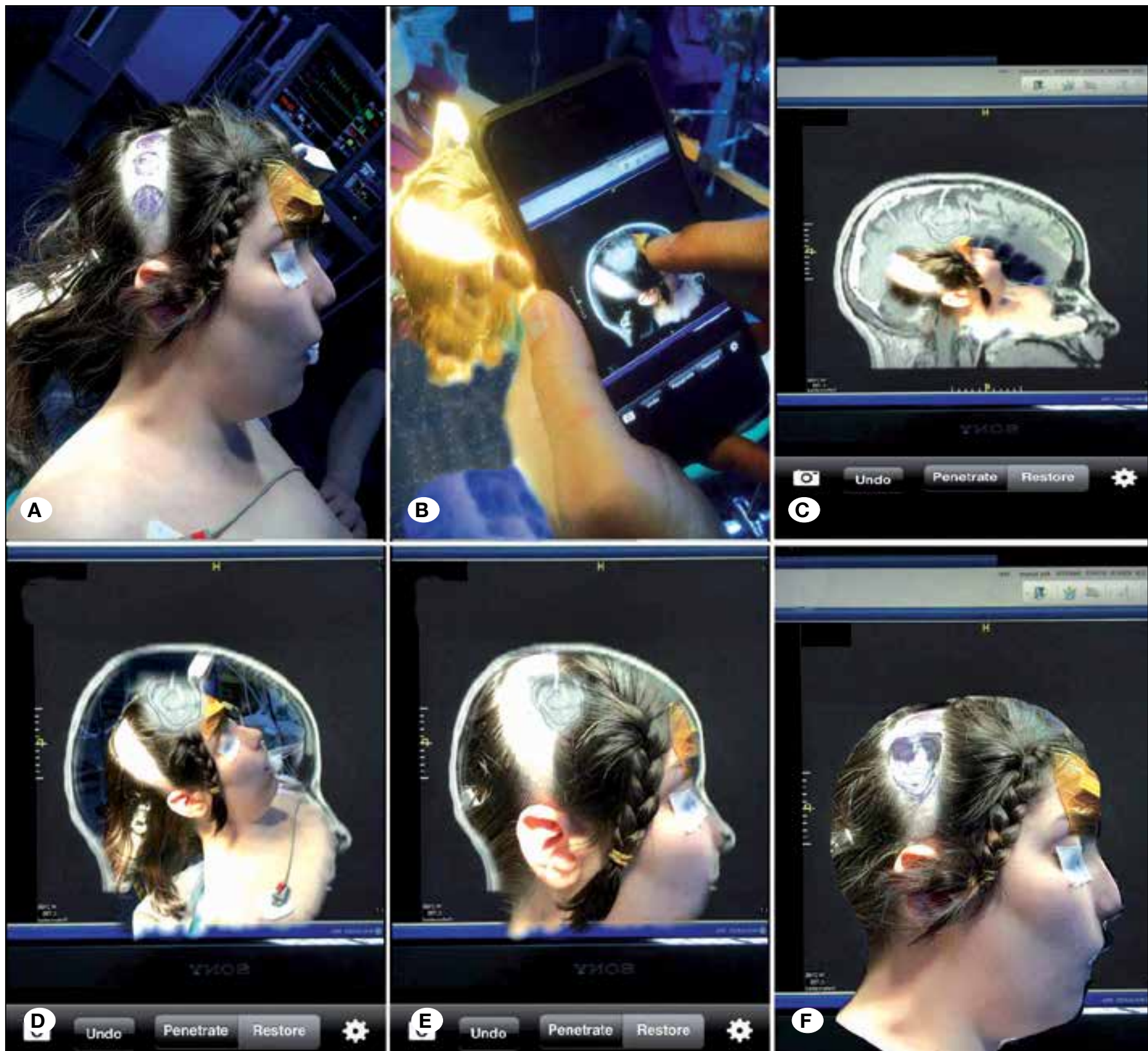

Figure 2: Surgical position of patient (A). Using the See-Through Camera app to erase the area on the smartphone display bordering the head contour by finger touch, excluding the tumor (B). Visualization of patient's head through the erased area and merging the real-time camera picture of the patient with the existing main image (C-E). Final image showing the tumor image superimposed over the patient's scalp (F). 
unit. The scoring system was based on the extent to which the tumor image on smartphone screen was superimposed on the tumor projection on the scalp that was estimated by the navigation system. If the tumor image on the smartphone display fits the on-screen tumor figure derived by the navigation system by $80 \%-100 \%$, a maximum score of 5 points was given. For every $20 \%$ reduction in superposition, the score was decreased by 1 point, reaching 0 for no correspondence.

\section{RESULTS}

Marking the tumor in each sagittal section on monitor display, transferring the images to the app, re-configuring the main image, and fusing the camera image of patient with the edited radiological image consisting of only the tumor took approximately $10 \mathrm{~min}$. According to our scoring system, five patients scored 5 points, seven patients scored 4 points, four patients scored 3 points, one patient scored 2 points, two patients scored 1 point and one patient scores 0 points. All the surgical procedures were guided by the microscope-integrated neuronavigation system, and all were successful, with complete resection of the tumors. The patient characteristics and app performance scores for each procedure are shown in Table I.

\section{DISCUSSION}

Microscope-integrated neuronavigation systems are used in almost all neurosurgical procedures. Preoperative visualization of lesions and determination of tumor location by microscopy improves surgeon comfort and subsequent surgical outcomes. With an increasing rate of usage of portable communication devices in recent decades, software developers have worked hard to make hand-held computers smarter and increasingly better adapted to daily and business-related activities (19). Medical software developers comprise a small subcategory of the smartphone app industry. Compared with other categories, these apps are less popular and are less developed $(16,17)$. Although there are thousands of medical apps for Apple, Android, Google and Blackberry operating systems, the number of effective and convenient apps used in medical decision-making and stage implementation is still less than expected. Additionally, apps used for surgery and approved as a surgical device applied to invasive procedures still do not exist. Although there are radiological image viewer apps for portable computers used as microscope displays, they are not considered medical or surgical devices (20).

In July 2011, the United States Food and Drug Administration classified mobile medical applications as apps on a

Table I: General Characteristics of Patients, Pathologies and Superposition Ratios of Images' Distribution according to Patients

\begin{tabular}{cccccc}
\hline Patient & Gender/Age (years) & Size & Location & Pathology & SRI $/$ Point \\
\hline $\mathbf{1}$ & M/38 & $2 \mathrm{~cm}$ & Parietal & Grade II Glial Tm & 1 \\
\hline $\mathbf{2}$ & $\mathrm{M} / 24$ & $8 \mathrm{~cm}$ & Parietal & Meningioma & 5 \\
\hline $\mathbf{3}$ & $\mathrm{F} / 36$ & $4 \mathrm{~cm}$ & Frontal & Meningioma & 3 \\
\hline $\mathbf{4}$ & $\mathrm{M} / 53$ & $3 \mathrm{~cm}$ & Parietal & Grade IV Glial Tm & 4 \\
\hline $\mathbf{6}$ & $\mathrm{M} / 48$ & $7 \mathrm{~cm}$ & Parietal & Meningioma & 5 \\
\hline $\mathbf{7}$ & $\mathrm{F} / 5$ & $2 \mathrm{~cm}$ & Temporal & Abscess & 0 \\
\hline $\mathbf{8}$ & $\mathrm{F} / 65$ & $4 \mathrm{~cm}$ & Frontal & Metastasis & 5 \\
\hline $\mathbf{9}$ & $\mathrm{F} / 54$ & $3 \mathrm{~cm}$ & Frontal & Meningioma & 3 \\
\hline $\mathbf{1 0}$ & $\mathrm{M} / 58$ & $6 \mathrm{~cm}$ & Parietal & Meningioma & 4 \\
\hline $\mathbf{1 1}$ & $\mathrm{F} / 69$ & $3 \mathrm{~cm}$ & Parietal & Metastasis & 2 \\
\hline $\mathbf{1 2}$ & $\mathrm{F} / 12$ & $2.5 \mathrm{~cm}$ & Occipital & Abscess & 1 \\
\hline $\mathbf{1 3}$ & $\mathrm{M} / 38$ & $6 \mathrm{~cm}$ & Parietal & Grade IV Glial Tm & 5 \\
\hline $\mathbf{1 4}$ & $\mathrm{F} / 45$ & $3.5 \mathrm{~cm}$ & Parietal & Metastasis & 3 \\
\hline $\mathbf{1 5}$ & $\mathrm{M} / 56$ & $4 \mathrm{~cm}$ & Frontal & Meningioma & 3 \\
\hline $\mathbf{1 6}$ & $\mathrm{F} / 41$ & $3 \mathrm{~cm}$ & Parietal & Metastasis & 4 \\
\hline $\mathbf{1 7}$ & $\mathrm{F} / 38$ & $4 \mathrm{~cm}$ & Parietal & Meningioma & 4 \\
\hline $\mathbf{1 8}$ & $\mathrm{F} / 42$ & $4.5 \mathrm{~cm}$ & Parietal & Meningioma & 4 \\
\hline $\mathbf{1 9}$ & $\mathrm{M} / 64$ & $3 \mathrm{~cm}$ & Frontal & Metastasis & 2 \\
\hline $\mathbf{2 0}$ & $\mathrm{F} / 58$ & $3.5 \mathrm{~cm}$ & Parietal & Grade IV Glial Tm & 4 \\
\hline $\mathbf{1 0}$ & $\mathrm{M} / 20$ & $5 \mathrm{~cm}$ & Parietal & Meningioma & 5 \\
\hline
\end{tabular}

SRI: The superposition ratio of the tumor image, M: Male, F: Female, Tm: Tumor. 
wireless device that are accessories of medical devices, apps converting a mobile platform to a medical device, or apps that include patient data used to guide physicians $(1,15)$. In January 2016 , the names of 80 apps included in medicine category were retrieved when the search word was 'neurosurgery'. Most of those apps were educational, and were developed by professional organizations or for academic meetings, easy access to journal official websites or free books. Some apps were designed to guide neurosurgeons in disease/disorder management and surgical decision-making. Others were 3-dimensional neuro-anatomic animations, or useful in applying grading and classification systems or treatment guidelines. None of the available apps could be used in any part of a surgical procedure. Although some attachable medical hardware is compatible with smartphones or tablet computers working with their own specific apps (e.g., sphygmomanometers, blood glucometers), no portable hardware-software combination is currently approved for use in invasive procedures. Although See-Through Camera is a photography application not designed for medical use and not approved for use in any part of a surgical procedure; it is instructional in suggesting how to creating future pocket navigation systems and might serve as the basis of such a unit.

The primary aim of the study was to determine whether an app could be applied to neurosurgical procedures and used at any surgical stage. As stressed above, none of the surgical procedures was planned using this app, but its usefulness and accuracy in finding the location of tumor were tested in
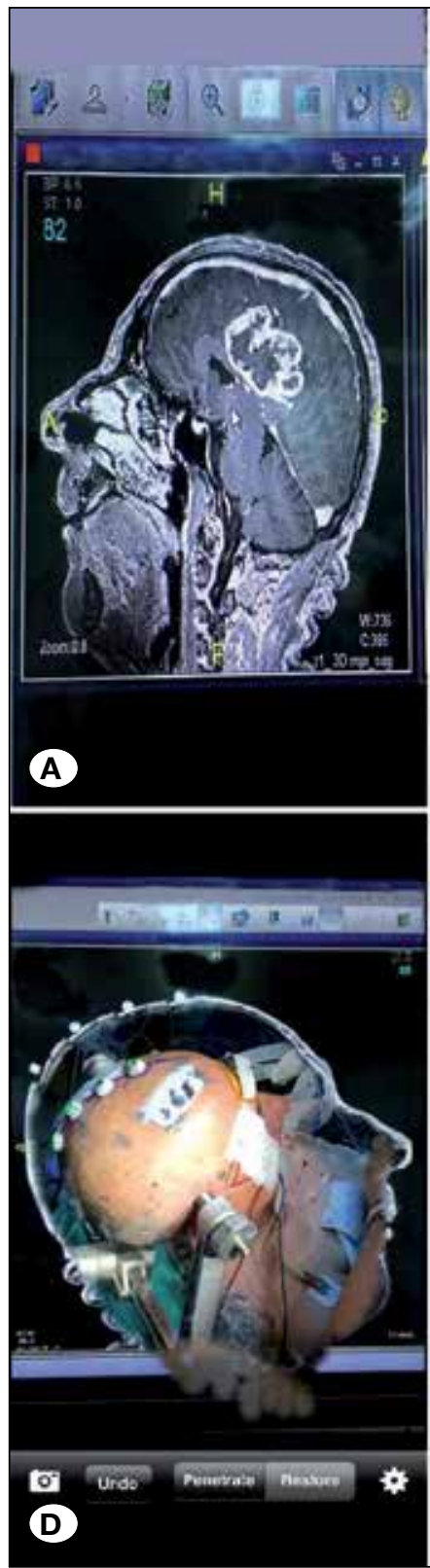
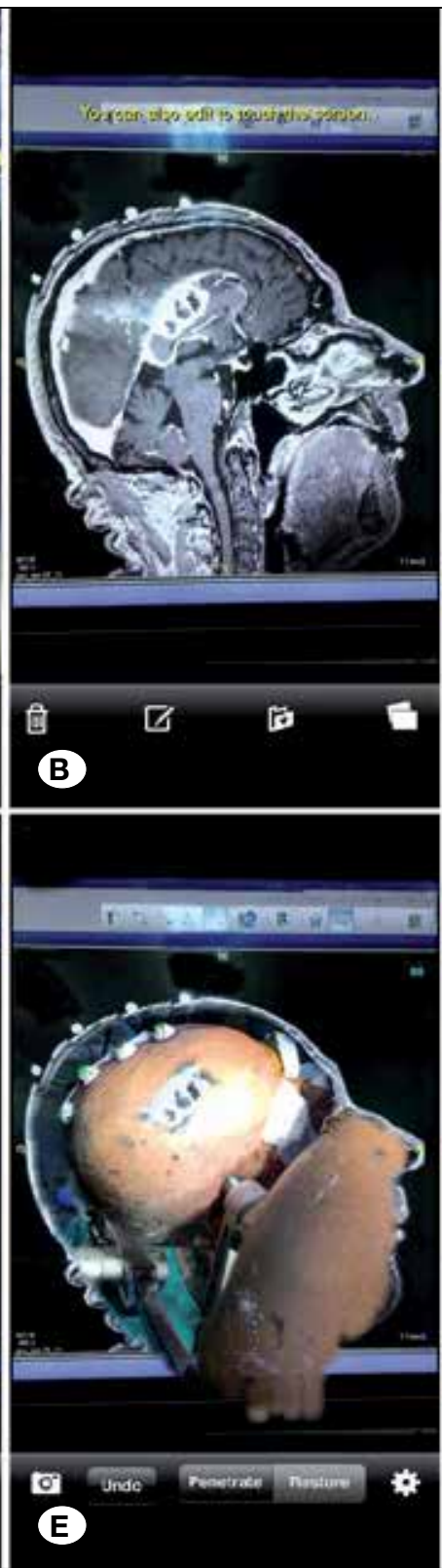
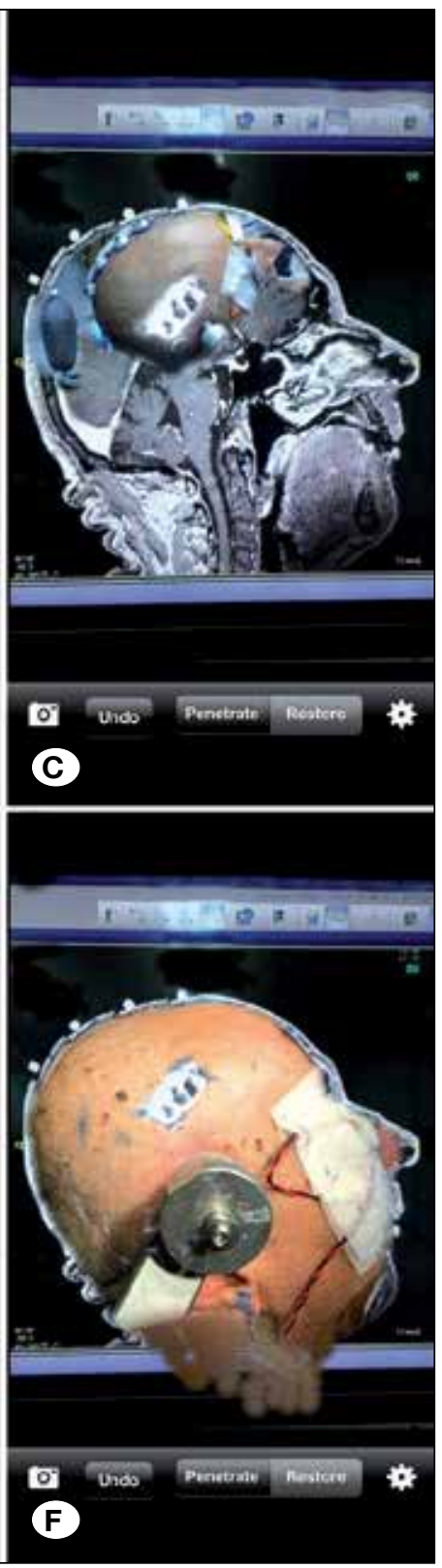

Figure 3: Stages of surgical planning of a patient with a midline tumor using the SeeThrough Camera app. Main image of the patient (A). Mirror image of the main image (B). Stepwise visualization of patient inside the transparent region on display (C-E). Contour fitting of two images (F). It should be noted that calvarial artificial markers increase the sensitivity of the application and allow more accurate superposition of the two images. 
comparison with data obtained using a BrainLab navigation system. Superposition of images was distorted with decrease in tumor size and localization decreased in accuracy with distance from the centre of lateral convexity of the skull. Contour fitting of the main image and that of the patient's head involves superposition of transparent tumor and scalp images. For optimal image fusion, the outlines of the images should be clearly distinguishable and the image contours should be regular. Some circumstances such as presence of hair or poor resolution of the scalp surface image may cause failure in merging of two pictures. Placing additional MRcompatible on the scalp may facilitate image matching (Figure $3 \mathrm{~A}-\mathrm{F})$. Although, no case of midline cerebral pathology was included in this study, and the accuracy and compatibility of the app should be evaluated in such patients, it may be effective in finding an access route to tumors located in the midline (Figure $3 \mathrm{~A}-\mathrm{F}$ ). See-Through Camera may also have neuro-anatomy applications (Figure $4 \mathrm{~A}-\mathrm{C}$ ). Comparison and examination of the location of deep and superficial brain structures with each other and with radiographic visualizations of these neuro-anatomic regions in a single picture may be feasible. The app may also be useful for preparation of neuroanatomic studies and presentations, and illustration of the anatomic relationships of brain structures in instructional figures.

Only one medical app designed for localization of supratentorial lesions prior to surgery was found. The app, Sina Neurosurgical Assist, is used to assist image-based scalp localization, and uses the same photography technique as See-Through Camera $(4,5)$. Both apps superimpose a transparent photograph over a real-time camera image. Sina is an Android app available at the Google Play Store (https:// play.google.com/store/apps/details?id=sina.neurosurgical. assist\&hl=en), and See-Through Camera is an iOS app that can be downloaded from the Apple Store. An advantage of
See-Through Camera is that the adjustable transparency of the background picture (Figure 3B). Also, other useful options of the iOS app are ability to change the editing mode, pen size, camera resolution. The camera input provides a muchappreciated service for practitioners.

There are professional drawing or illustration apps designed for computers that allow merging of multiple pictures as multiple layers with several opacities. Compared with comprehensive drawing software, the ability to merge only two image layers may seem to be a disadvantage, but this smartphone/tablet app has the advantage of practicality. Smartphones and tablets may be preferable for use in surgical procedures because of their portability and size. Apps provide extra functionality to these devices and broaden their use in every professional sector. In the future, these devices may be developed as miniaturized neuronavigation systems. Integrated laser probe accessories may map the patient's face and combining those with MR images may be able to create a 3-dimensional image of the patient and the pathology.

\section{- CONCLUSION}

The See-Through Camera app was found to have potential applicability to surgical practice and anatomical study. The app has not been approved for medical use, but may be used to add value to and facilitate medical training and training in anatomy. In the future, cooperation of software and hardware developers and health care professionals may give rise to portable neuronavigation systems and microscopes small enough to be carried in surgeons' pockets. The concept described in this report may sensitize neurosurgeons to this improving and expanding technology and may lead them to create convenient and comprehensive apps in surgical practice.
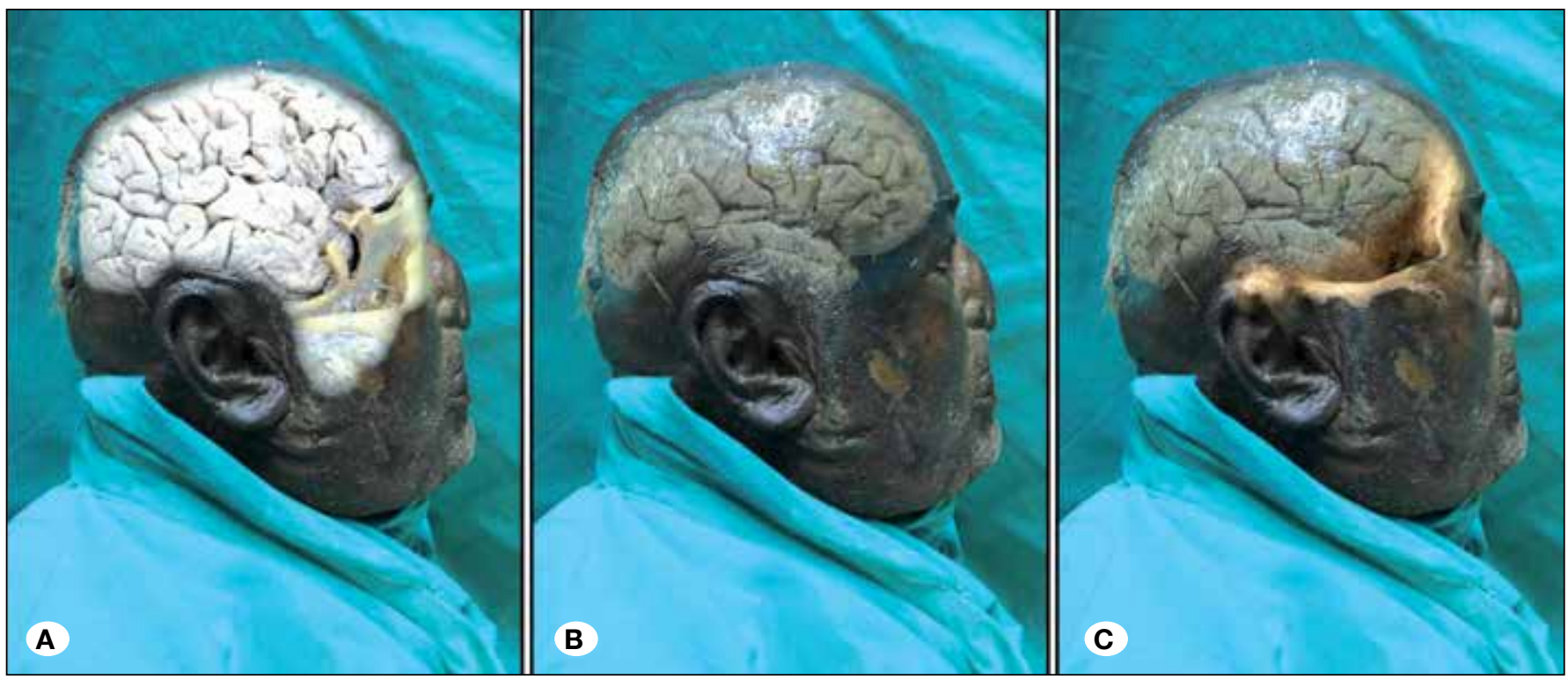

Figure 4: An example showing using of See-Through App for anatomic study. Merging of 2 images without transparency (A). Merging of semi-transparent head with brain (B). Merging of 3 photos including brain, calvarium and scalp as one image (C). 


\section{ACKNOWLEDGEMENT}

Preparation for publication of this article was partly supported by the Turkish Neurosurgical Society.

\section{REFERENCES}

1. Barton AJ: The regulation of mobile health applications. BMC Med 10:46, 2012

2. Cenydd LA, John NW, Phillips NI, Gray WP: VCath: A tabletbased neurosurgery training tool. Stud Health Technol Inform 184:20-23, 2013

3. Chang AY, Ghose S, Littman-Quinn R, Anolik RB, Kyer A, Mazhani L, Seymour AK, Kovarik CL: Use of mobile learning by resident physicians in Botswana. Telemed $\mathrm{J} \mathrm{E}$ Health 18: 11-13, 2012

4. Eftekhar B: A smartphone app to assist scalp localization of superficial supratentorial lesions-technical note. World Neurosurg 85:359-363, 2016

5. Eftekhar B: App-assisted external ventricular drain insertion. J Neurosurg 125:754-758, 2016

6. Franko OI: Smartphone apps for orthopaedic surgeons. Clin Orthop Relat Res 469: 2042-2048, 2011

7. Franko OI, Tirrell TF: Smartphone app use among medical providers in ACGME training programs. J Med Syst 36:31353139, 2012

8. Freshwater MF: iPhone and iPad applications for plastic surgeons. J Plast Reconstr Aesthet Surg 64:1397-1399, 2011

9. Jacquot F, Charpentier A, Khelifi S, Gastambide D, Rigal $R$, Sautet A: Measuring the Cobb angle with the iPhone in kyphoses: A reliability study. Int Orthop 36:1655-1660, 2012

10. Kahn JG, Yang JS, Kahn JS: 'Mobile' health needs and opportunities in developing countries. Health Aff (Millwood) 29:252-258, 2010
11. Kubben P: Neuromind 2: Interactive decision support for neurosurgery. Surg Neurol Int 3:109, 2012

12. Kubben PL: Neurosurgical content for mobile devices. Surg Neurol Int 1:46, 2010

13. Mobasheri $M H$, Johnston $M$, King $D$, Leff $D$, Thiruchelvam P, Darzi A: Smartphone breast applications - what's the evidence? Breast 23:683-689, 2014

14. Mobasheri $M H$, Johnston $M$, Syed UM, King D, Darzi $A$ : The uses of smartphones and tablet devices in surgery: A systematic review of the literature. Surgery 158:1352-1371, 2015

15. Mobile Medical Applications: Guidance for Industry and Food and Drug Administration Staff. Document issued on February 9, 2015. Available at: http://www.fda.gov/downloads/medicaldevices/deviceregulationandguidance/guidancedocuments/ucm263366.pdf

16. Mosa AS, Yoo I, Sheets L: A systematic review of healthcare applications for smartphones. BMC Med Inform Decis Mak 12:67, 2012

17. Payne KB, Wharrad H, Watts K: Smartphone and medical related App use among medical students and junior doctors in the United Kingdom (UK): A regional survey. BMC Med Inform Decis Mak 12:121, 2012

18. Research2Guidance. The mobile health global market report 2013 - 2017 (Vol.3). The commercialization of the mHealth applications Graphical package. March 2013. Available at:http:// www.research2guidance.com/shop/index.php/downloadable/sample/sample_id/262/.

19. Seabrook HJ, Stromer JN, Shevkenek C, Bharwani A, de Grood J, Ghali WA: Medical applications: A database and characterization of apps in Apple iOS and Android platforms. BMC Res Notes 7:573, 2014

20. Soehngen E, Rahmah NN, Kakizawa Y, Horiuchi T, Fujii Y, Kiuchi T, Hongo K: Operation-microscope-mounted touch display tablet computer for intraoperative imaging visualization. World Neurosurg 77:381-383, 2012 